\title{
The burden of cardiovascular risk factors among seniors with congenital heart disease: A single tertiary center experience
}

\author{
Anna Kwiatek-Wrzosek, Ewa Kowalik, Mirosław Kowalski, Piotr Hoffman \\ Department of Congenital Heart Diseases, National Institute of Cardiology, Warszawa, Poland
}

\author{
Correspondence to: \\ Anna Kwiatek-Wrzosek, MD, \\ Department of Congenital Heart \\ Diseases, \\ National Institute of Cardiology, \\ Alpejska 42, 04-628 Warszawa, \\ Poland, \\ phone: +48223434 400, \\ e-mail: akwiatek@ikard.pl \\ Copyright by the Author(s), 2021 \\ Kardiol Pol. 2021: \\ 79 (11): 1251-1255; \\ DOI: 10.33963/KP.a2021.0129 \\ Received: \\ July 13, 2021 \\ Revision accepted: \\ October 8, 2021 \\ Published online: \\ October 8, 2021
}

\section{A B S TR A C T}

Background: The number of adults with congenital heart disease (ACHD) surviving to old age is increasing worldwide. Acquired cardiovascular comorbidities may complicate the course and treatment of the underlying congenital disease and worsen the prognosis.

Aims: The study aimed to assess the burden of cardiovascular (CV) risk factors among elderly patients with ACHD.

Methods: A retrospective analysis of data on all patients $\geq 60$ years of age hospitalized in a tertiary clinic for ACHD was performed from July 2013 to March 2020. We collected information on smoking status, body mass index, and the presence of dyslipidemia, systemic hypertension, and diabetes.

Results: The most common CV risk factors among 322 patients $\geq 60$ years of age (median age 66 years; 34\% men) were: being overweight/obesity (65.5\%), dyslipidemia (64.9\%), and arterial hypertension (60.6\%). Over $21 \%$ of patients suffered from diabetes, and $25.8 \%$ were smokers. Over $54 \%$ of patients had two or $3 \mathrm{CV}$ risk factors. Patients above 70 years of age were healthier in terms of being overweight/obesity, dyslipidemia, and smoking status. Patients with mild ACHD were more likely hypertensive compared to individuals with complex defects. The highest $\mathrm{CV}$ burden was noted in younger men with mild ACHD.

Conclusions: We demonstrated a high burden of CV risk factors in seniors with ACHD. Special attention should be paid to the identification and control of classical CV risk factors in order to prevent acquired CV disease in this population.

Key words: aging, cardiovascular risk factors, congenital heart disease, seniors

Kardiol Pol 2021; 79, 11: 1251-1255

\section{INTRODUCTION}

The enormous progress in pediatric cardiology and cardiac surgery has resulted in a constantly growing population of adults with congenital heart disease (ACHD), and an increasing number of these are $\geq 60$ years of age. They constitute an entirely new cohort of patients who require particular attention from care providers. This population includes not only patients with simple lesions but also individuals with heart defects of at least moderate severity [1]. According to the extrapolation to the European Union countries presented by Baumgartner et al. [2], the percentage of ACHD of 60 years and older may reach $11 \%$ of all ACHD patients by 2030 . Studies concerning adolescents and younger adults showed that a low proportion of individuals with ACHD are not exposed to classical risk factors of acquired cardiovascular diseases $[3,4]$. Those diseases complicate the course and treatment of the underlying congenital disease and increase resource consumption [5]. Thus, cardiovascular (CV) risk factors and age-related acquired cardiovascular lesions are identified as a new factor in the older ACHD population with a possible impact on the prognosis and quality of life. Previously published data regarding geriatric ACHD cohorts either did not report details on CV risk factors [1], had some methodological limitations [6], or reported data on small, predefined subgroups [7]. Thus, 
WHAT'S NEW?

Adult patients with congenital heart disease (ACHD) have longer life expectancy due to better medical care, but data regarding the prevalence of classical cardiovascular (CV) risk factors are scarce. We examined the burden of CV risk factors in ACHD patients $\geq 60$ years of age. Among 322 patients in our study, the most common CV risk factors were: being overweight/obesity (65.5\%), dyslipidemia (64.9\%), and arterial hypertension (60.6\%). Over $21 \%$ of patients suffered from diabetes, and $25.8 \%$ were smokers. Over $54 \%$ of patients had two or three CV risk factors. Patients above 70 years of age were healthier in terms of being overweight/obesity, dyslipidemia, and smoking status. The highest CV burden was recorded in younger men with mild ACHD. Our findings emphasize the importance of preventive medicine in this population. Therefore, identification and control of CV risk factors should be a part of standard medical care in the elderly with ACHD.

this study aimed to assess the occurrence of $C V$ risk factors in patients with ACHD beyond the age of 60 in a population of a tertiary center for ACHD in Poland.

\section{METHODS}

A retrospective analysis of patients hospitalized in our department between July 2013 and March 2020 was performed. The causes of hospitalization were: (1) newly diagnosed ACHD; (2) planned diagnostic procedures or elective surgical or percutaneous treatment; (3) emergency cases; (4) routine check-up in patients with severe ACHD, ACHD-related pulmonary arterial hypertension, or in older ACHD patients (one-day hospitalization). The inclusion criteria were: (1) age $\geq 60$ years at the time of hospitalization; (2) previously or newly diagnosed congenital heart disease. Congenital heart defects were classified as mild, moderate, or severe according to the European Society of Cardiology guidelines [8]. Data on body mass index (BMI), the presence of dyslipidemia, systemic hypertension, diabetes, and the history of smoking were collected.

Patients were defined as hypertensive if they were on antihypertensive treatment, or they were newly diagnosed with arterial hypertension during the hospitalization according to the European Society of Cardiology hypertension guidelines [9].

Patients with low-density lipoprotein cholesterol concentrations (obtained by Friedwald formula unless plasma triglyceride concentration exceeded $400 \mathrm{mg} / \mathrm{dl}$ ) above target levels recommended in the European Society of Cardiology guidelines on cardiovascular disease prevention in clinical practice [10], or with a previous diagnosis of dyslipidemia, or receiving lipid-lowering therapy were classified as having dyslipidemia. Patients with diabetes (type 2 or type 1) had a documented disease managed with diet, oral hypoglycemic medications, or insulin, or they were newly diagnosed with diabetes (according to American Diabetes Association criteria [11]) during the index hospitalization. Being overweight was defined as BMI $25-29.9 \mathrm{~kg} / \mathrm{m}^{2}$ and obesity as $B M I \geq 30 \mathrm{~kg} / \mathrm{m}^{2}$ [12]. Patients self-reported their health behaviors regarding cigarette smoking (non-smoker, previous or current smoker). Patients were classified as having coronary artery disease (CAD) when they had a history of myocardial infarction or coronary artery bypass surgery, or they had angiographically proven CAD. If a patient was admitted to our clinic more than once during the given timeframe, data from the first hospitalization were taken into account. The study was approved by the institutional medical ethics committee.

\section{Statistical analysis}

Data were presented as the median and interquartile range (IQR) and categorical variables were expressed as numbers and frequencies. The Shapiro-Wilk test was used to assess the normality of variable distributions. The comparisons of differences between groups were performed using the Mann-Whitney $U$ test, and in the case of categorical variables, the Z-test was used. A two-sided $P$-value of $<0.05$ was considered statistically significant. All data were analyzed with $\mathrm{R}$ software package version 3.2.4.

\section{RESULTS}

We identified 322 patients with ACHD who were $\geq 60$ years of age at any hospital stay in our clinic during the study period (median age, 66.0; range, 60-87 years). Most individuals had simple cardiac defects, however, there were also 14 patients with severe lesions ( 8 with congenitally corrected transposition of the great arteries, 5 with Eisenmenger syndrome, and one patient with repaired double-outlet right ventricle). More detailed information regarding baseline characteristics is provided in Table 1.

Being overweight/obesity and dyslipidemia were the most common CV risk factors followed by systemic arterial hypertension. Every fifth patient suffered from diabetes and every fourth was a current or previous smoker (Table 1).

In the majority of patients (over $54 \%$ ), 2 or 3 CV risk factors were recorded. Only 18 patients had no CV risk factors (Figure 1).

Among men, there were more smokers and individuals with systemic arterial hypertension when compared to women (Table 2). Patients above and below 70 years of age differed significantly in terms of being overweight/obesity, dyslipidemia, and smoking status (Table 2).

Patients with mild ACHD were more likely to be hypertensive compared to patients with more complex lesions. The trend towards a higher number of smokers among mild ACHD patients was also observed. The preva- 
Table 1. Clinical characteristics and prevalence of cardiovascular risk factors among 322 ACHD seniors

\begin{tabular}{lc}
\multicolumn{1}{c|}{ Clinical characteristics } \\
Age, years & $66(62-69)$ \\
Age structure & $242(75.51)$ \\
$60-69$ years, $\mathrm{n}(\%)$ & $68(21.11)$ \\
$70-79$ years, $\mathrm{n}(\%)$ & $12(3.73)$ \\
$\geq 80$ years, $\mathrm{n}(\%)$ & $110(34.2)$ \\
Men, $\mathrm{n}(\%)$ & \\
ACHD complexity & $263(81.42)$ \\
Mild, $\mathrm{n}(\%)$ & $45(13.93)$ \\
Moderate, $\mathrm{n}(\%)$ & $14(4.33)$ \\
Severe, $\mathrm{n}(\%)$ & $26.8(24.0-30.1)$ \\
BMl, kg/m ${ }^{2}$ & \\
Cardiovascular risk factors & $2(1-3)$ \\
Number of risk factors & $211(65.53)$ \\
Overweight/obesity, $\mathrm{n}(\%)$ & $195(60.56)$ \\
Arterial hypertension, $\mathrm{n}(\%)$ & $209(64.91)$ \\
Dyslipidemia, $\mathrm{n}$ (\%) & $69(21.43)$ \\
Diabetes, $\mathrm{n}(\%)$ & $83(25.78)$ \\
Smoking, $\mathrm{n}(\%)$ & \\
\hline
\end{tabular}

Data are presented as the median (interquartile range [IQR]) or number (\%) Abbreviations: ACHD, adult congenital heart disease; BMI, body mass index

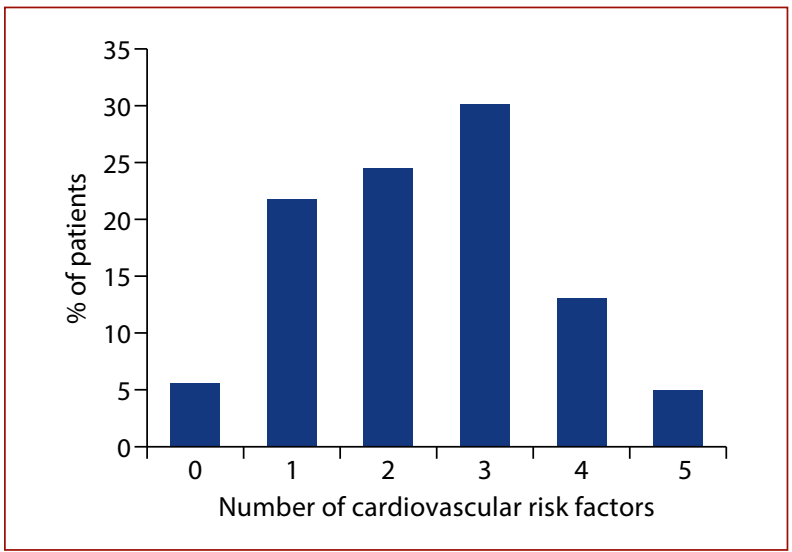

Figure 1. Number of cardiovascular risk factors in adult congenital heart disease patients aged $\geq 60$ years lence of diabetes did not differ significantly in all analyzed subgroups. The younger (60-69 years old) men with mild ACHD had the highest burden of cardiovascular risk factors (Table 3). Angiographically confirmed atherosclerotic CAD was found in $68(21 \%)$ patients.

\section{DISCUSSION}

The number of individuals with ACHD surviving to old age is increasing and they are exposed to classical CV risk factors and acquired heart disease as the general population. We observed that in most patients with ACHD aged $\geq 60$ years at least two CV risk factors are present with the highest prevalence of risk factors found among younger (60-69 years old) men with mild heart defects.

The most common CV risk factor was being overweight and obesity, which affected over $65 \%$ of the study population. Our findings are like those previously reported in younger (mean age, 52 years) ACHD patients in the United States of America [13]. In the study by Lerman et al. [13] 59.5\% of ACHD patients were overweight or even morbidly obese, and the prevalence of being overweight/obesity was similar when compared to the general population. Notwithstanding, in our study, the problem of being overweight and obesity in seniors with ACHD is less common than in the general Polish population [14]. Likewise, the authors of WOBASZ II study reported a smaller number of overweight/obese individuals of advanced age ( $\geq 75$ years).

Dyslipidemia was a common cardiovascular risk factor as being overweight/obesity (almost 65\%). Contrary to our data, Afilalo et al. [6] reported that among geriatric patients with ACHD in Canada only $27 \%$ had been diagnosed with dyslipidemia. A recently published smaller Canadian study on seniors with moderate to complex ACHD [7] also showed a lower prevalence of dyslipidemia (39\%) when compared to our results (56\% in the subgroup with moderate/severe ACHD).

Table 2. Cardiovascular risk factors according to age, gender, and ACHD complexity

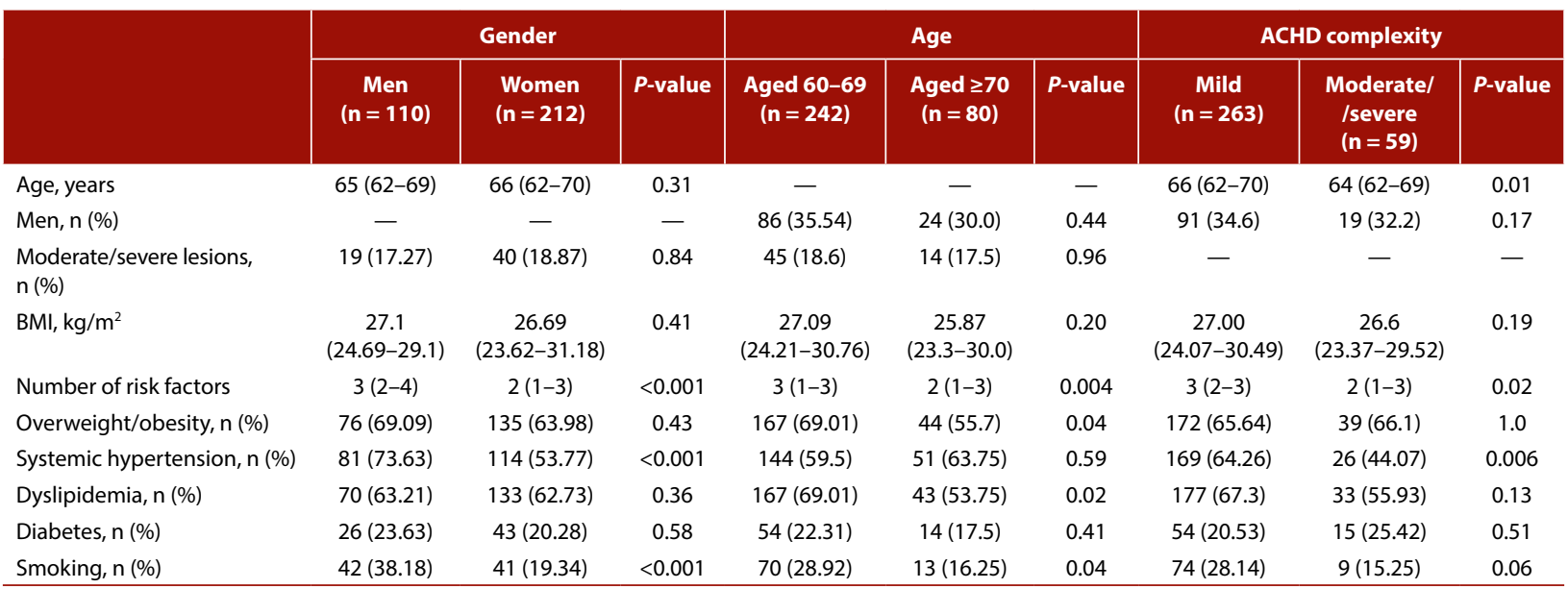

Data are presented as the median (interquartile range [IQR]) or number (\%)

Abbreviations: see Table 1 
Table 3. Cardiovascular risk factors in younger men with mild heart defects compared to other ACHD patients

\begin{tabular}{|c|c|c|c|}
\hline & $\begin{array}{l}\text { Men 60-69 years old, with mild ACHD } \\
\qquad n=70\end{array}$ & $\begin{array}{l}\text { Rest of the study group } \\
\qquad n=252\end{array}$ & P-value \\
\hline Number of risk factors, median (IQR) & $3(2-4)$ & $2(1-3)$ & $<0.001$ \\
\hline Overweight/obesity, n (\%) & $54(77.14)$ & $157(62.55)$ & 0.03 \\
\hline Systemic hypertension, n (\%) & $55(78.57)$ & $140(55.55)$ & $<0.001$ \\
\hline Dyslipidemia, n (\%), & $52(74.28)$ & $158(62.7)$ & 0.10 \\
\hline Diabetes, n (\%) & $19(27.14)$ & $50(19.84)$ & 0.25 \\
\hline Smoking, n (\%) & $31(44.28)$ & $52(20.63)$ & $<0.001$ \\
\hline
\end{tabular}

Abbreviations: IQR, interquartile range; other — see Table 1

We observed a higher overall prevalence of arterial hypertension in the geriatric ACHD population compared to the Canadian study by Afilalo et al. (60.6\% vs. $47 \%$, respectively) [6]. Moreover, the authors did not find any significant differences in the prevalence of arterial hypertension according to the complexity of heart defects, whereas in our population patients with mild anomalies were diagnosed with systemic hypertension more often than individuals with moderate/severe heart defects. On the other hand, the prevalence of systemic hypertension among the elderly with moderate/complex ACHD in the study by Reich et al. [7] was higher (54\%) than in our report among individuals with moderate/severe disease (44\%).

Every fourth person in our study group was a current or previous tobacco smoker with a significant predominance of men and younger individuals. In a large study including younger ACHD patients from 15 countries (over 4000 participants, median age, 32 years) fewer patients ( $14 \%$ of men and $11 \%$ of women) smoked cigarettes in contrast to our results [15]. When compared to Switzerland, the country with the highest number of smokers among ACHD patients in the above-mentioned study ( $24 \%$ of men, $19 \%$ of women), the use of tobacco was higher in men but not in women in our elder ACHD population. Similarly, Moons et al. [15] reported that individuals with mild ACHD smoke cigarettes more often than people with moderate to severe defects. Interestingly, in patients with moderate to complex ACHD over the age of 60 years in Southern Alberta (Canada), the percentage of smokers was twice as high as in our study [7].

Twenty-one percent of ACHD patients in our study suffered from diabetes. The prevalence of diabetes is higher than in seniors in the general population of Poland according to the Polish national PolSenior study [16]. This difference might be explained by the findings by Madsen et al. [17], who showed that CHD survivors in Denmark were at a substantially increased risk of developing diabetes mellitus type 2 after the age of 30 compared with the general Danish population. On the other hand, in our study, the percentage of diabetic patients was higher than in the geriatric CHD population in Quebec/Canada (16\%) and South Alberta (13\%) [6, 7]. As diabetes is a risk factor for arrhythmias in ACHD [18] and it was identified as an independent predictor of all-cause mortality [6], its occurrence among ACHD survivors in Poland deserves special attention.

Our study shows that among elderly patients with ACHD the incidence of modifiable cardiovascular risk factors is significantly higher in younger men (60-69 years) with mild defects. Such an observation has not been reported yet. One might speculate that patients with ACHD who live a healthier lifestyle are more likely to survive beyond the age of 70 .

The results of our study convey several important findings. It clearly shows that classical CV risk factors are often encountered in older ACHD patients. Owing their adverse impact on prognosis in general population we need to incorporate all accepted measures to detect and control them into our regular clinical practice while dealing with ACHD patients. Also, further studies are highly justified to elucidate reasons for such high prevalence of CV risk factors among ACHD patients, whose life expectancy is lower than in general population due to primary disease. Another important issue is related to the pharmacotherapy of acquired CV disease accompanying congenital cardiac defects. Some of these medications ( $\beta$-blockers, calcium channel blockers, angiotensin-converting enzyme inhibitors or sartans) may interfere with abnormal hemodynamics caused by a congenital abnormality. As we learned from studies dedicated to heart failure in ACHD population, guidelines-based pharmacotherapy in this specific group may be less effective or even potentially harmful. Therefore, the implementation of the treatment requires meticulous clinical monitoring.

\section{Study limitations}

We are aware of some methodological limitations of our study. First, the character of the study conducted in a single tertiary center may influence the results. Furthermore, tobacco smoking was classified according to the patient's information, thus its prevalence may be underestimated. Also, we were not able to directly compare the incidence of $\mathrm{CV}$ risk factors in ACHD and non-ACHD seniors for methodological reasons. We did not analyze psychosocial and economic factors which can also influence cardiovascular risk [19]. The prevalence of CAD might be underestimated as only patients with suspected CAD and individuals undergoing cardiac surgery were investigated. We did 
not perform post hoc power analysis for subgroups; thus these results should be interpreted with caution. Finally, the cross-sectional design of the study did not allow us to assess the impact of cardiovascular risk factors on morbidity and mortality among elderly ACHD patients.

\section{CONCLUSIONS}

The study examined the burden of CV factors in elderly ACHD patients. Our findings stress the importance of proactive screening for classical CV risk factors in ACHD that should be controlled in order to reduce the prevalence of acquired comorbidities and worsening the overall prognosis in this population. Identification and treatment of classical CV risk factors should be part of standard medical care in seniors with ACHD.

\section{Article information}

Conflict of interests: None declared.

Open access: This article is available in open access under Creative Common Attribution-Non-Commercial-No Derivatives 4.0 International (CC BY-NC-ND 4.0) license, allowing to download articles and share them with others as long as they credit the authors and the publisher, but without permission to change them in any way or use them commercially. For commercial use, please contact the journal office at kardiologiapolska@ptkardio.pl.

How to cite: Kwiatek-Wrzosek A, Kowalik E, Kowalski M, Hoffman P.The burden of cardiovascular risk factors among seniors with congenital heart disease: A single tertiary center experience. Kardiol Pol. 2021; 79(11): 1251-1255, doi: 10.33963/KP.a2021.0129.

\section{REFERENCES}

1. Tutarel $\mathrm{O}$, Kempny A, Alonso-Gonzalez R, et al. Congenital heart disease beyond the age of 60: emergence of a new population with high resource utilization, high morbidity, and high mortality. Eur Heart J. 2014; 35(11): 725-732, doi: 10.1093/eurheartj/eht257, indexed in Pubmed: 23882067.

2. Baumgartner $\mathrm{H}$. Geriatric congenital heart disease: a new challenge in the care of adults with congenital heart disease? Eur Heart J. 2014; 35(11): 683-685, doi: 10.1093/eurheartj/eht358, indexed in Pubmed: 24014388.

3. Bauer UMM, Körten MA, Diller GP, et al. Cardiovascular risk factors in adults with congenital heart defects - recognised but not treated? An analysis of the German National Register for Congenital Heart Defects. Int J Cardiol. 2019; 277: 79-84, doi: 10.1016/j.ijcard.2018.08.009, indexed in Pubmed: 30100225.

4. Harris KC, Voss C, Rankin K, et al. Modifiable cardiovascular risk factors in adolescents and adults with congenital heart disease. Congenit Heart Dis. 2018; 13(4): 563-570, doi: 10.1111/chd.12612, indexed in Pubmed: 29938932.

5. Tutarel $\mathrm{O}$. Acquired heart conditions in adults with congenital heart disease: a growing problem. Heart. 2014; 100(17): 1317-1321, doi: 10.1136/heartjnl-2014-305575, indexed in Pubmed: 25099651.

6. Afilalo J, Therrien J, Pilote $\mathrm{L}$, et al. Geriatric congenital heart disease: burden of disease and predictors of mortality. J Am Coll Cardiol. 2011;
58(14): 1509-1515, doi: 10.1016/j.jacc.2011.06.041, indexed in Pubmed: 21939837.

7. Reich K, Moledina A, Kwan E, et al. Congenital heart disease (CHD) in seniors: a retrospective study defining a brand new cohort. Can Geriatr J. 2020; 23(4): 270-276, doi: 10.5770/cgj.23.435, indexed in Pubmed: 33282046.

8. Baumgartner H, De Backer J, Babu-Narayan SV, et al. ESC Scientific Document Group. 2020 ESC Guidelines for the management of adult congenital heart disease. Eur Heart J. 2021; 42(6): 563-645, doi: 10.1093/eurheartj/ehaa554, indexed in Pubmed: 32860028.

9. Williams B, Mancia G, Spiering W, et al. ESC Scientific Document Group . 2018 ESC/ESH Guidelines for the management of arterial hypertension. Eur Heart J. 2018; 39(33): 3021-3104, doi: 10.1093/eurheartj/ehy339, indexed in Pubmed: 30165516.

10. Piepoli MF, Hoes AW, Agewall S, et al. ESC Scientific Document Group. 2016 European Guidelines on cardiovascular disease prevention in clinical practice: the Sixth Joint Task Force of the European Society of Cardiology and Other Societies on Cardiovascular Disease Prevention in Clinical Practice (constituted by representatives of 10 societies and by invited experts) developed with the special contribution of the European Association for Cardiovascular Prevention \& Rehabilitation (EACPR). Eur Heart J. 2016;37(29):2315-2381, doi: 10.1093/eurheartj/ehw106, indexed in Pubmed: 27222591.

11. American Diabetes Association. Standards of medical care in diabetes - 2011. Diabetes Care. 2011; 34(Suppl 1): S11-S61, doi: 10.2337/dc11-S011, indexed in Pubmed: 21193625.

12. World Health Organization. Obesity: preventing and managing the global epidemic. WHO Technical Report. Series 894. Geneva, 2000

13. Lerman JB, Parness IA, Shenoy RU. Body weights in adults with congenital heart disease and the obesity frequency. Am J Cardiol. 2017; 119(4):638642, doi: 10.1016/j.amjcard.2016.10.050, indexed in Pubmed: 27931725.

14. Stepaniak U, Micek A, Waśkiewicz A, et al. Prevalence of general and abdominal obesity and overweight among adults in Poland. Results of the WOBASZ II study (2013-2014) and comparison with the WOBASZ study (2003-2005). Pol Arch Med Wewn. 2016; 126(9): 662-671, doi: 10.20452/pamw.3499, indexed in Pubmed: 27535012.

15. Moons P, Luyckx K, Kovacs AH, et al. APPROACH-IS Consortium and the International Society for Adult Congenital Heart Disease (ISACHD). Prevalence and effects of cigarette smoking, cannabis consumption, and co-use in adults from 15 countries with congenital heart disease. Can J Cardiol. 2019; 35(12): 1842-1850, doi: 10.1016/j.cjca.2019.07.635, indexed in Pubmed: 31813510.

16. Łabuz-Roszak B, Machowska-Majchrzak A, Skrzypek M, et al. Antiplatelet and anticoagulant therapy in elderly people with type 2 diabetes mellitus in Poland (based on the PolSenior Study). Arch Med Sci. 2017; 13(5): 10181024, doi: 10.5114/aoms.2017.68948, indexed in Pubmed: 28883841.

17. Madsen NL, Marino BS, Woo JG, et al. Congenital heart disease with and without cyanotic potential and the long-term risk of diabetes mellitus: a population-based follow-up study. J Am Heart Assoc. 2016; 5(7): e003076, doi: 10.1161/JAHA.115.003076, indexed in Pubmed: 27402234.

18. Cambronero-Cortinas E, Moratalla-Haro P, González-García AE, et al. Predictors of atrial tachyarrhythmias in adults with congenital heart disease. Kardiol Pol. 2020; 78(12): 1262-1270, doi: 10.33963/KP.15644, indexed in Pubmed: 33047943.

19. Bianco F, Bucciarelli V, Gallina S. The association between cardiovascular diseases and depressive symptoms in adults: the classic "chicken or egg" causality. Pol Arch Intern Med. 2021; 131(6): 497-498, doi: 10.20452/pamw.16038, indexed in Pubmed: 34184851. 